\title{
Equilibrium Joining Strategies of Delay-Sensitive Customers in a Queueing System with Service Quality Feedback
}

\author{
Peng Liu, ${ }^{1}$ Jun Lv, ${ }^{1}$ Tao Jiang $\mathbb{D}^{1},{ }^{1}$ and Xudong Chai ${ }^{2}$ \\ ${ }^{1}$ College of Economics and Management, Shandong University of Science and Technology, Qingdao 266590, China \\ ${ }^{2}$ School of Science, Nanjing University of Science and Technology, Nanjing 210094, China \\ Correspondence should be addressed to Tao Jiang; jtao0728@163.com
}

Received 6 November 2019; Revised 5 January 2020; Accepted 22 January 2020; Published 13 February 2020

Academic Editor: Xiaohua Ding

Copyright $\left({ }_{0} 2020\right.$ Peng Liu et al. This is an open access article distributed under the Creative Commons Attribution License, which permits unrestricted use, distribution, and reproduction in any medium, provided the original work is properly cited.

In some queueing systems, customers are frequently asked for giving a service quality feedback for their service at their service completion instants. Based on this phenomenon, in this paper, we model this type of queueing systems as clearing queues with service quality feedback and system maintenance. Once the system receives an unsatisfied (negative) feedback from customers (i.e., a customer is unsatisfied with the service), the system undergoes an adjustment procedure, and at the same time, all the present customers are forced to leave the system. By considering the waiting cost and reward, we discuss the joining behavior of customers and, respectively, derive the corresponding equilibrium joining strategies and social optimal strategies under different levels of information (the observable and the unobservable cases). Finally, some numerical examples are provided to show the effect of several system parameters on the equilibrium and optimal balking strategies.

\section{Introduction}

Nowadays, in some queueing systems such as manufacturing systems, restaurants, and computer network systems, we may encounter the following situation: all customers are forced to abandon the system due to the server receive a negative feedback, for example, in the context of a manufacturing system. A production process may consist of two phases: production phase and detection phase. The manufacturing system is equipped with a quality control detector for verifying the quality of the produced items at the second phase. If a finished product is detected to be defective, a reactive maintenance will be triggered, and all raw products are forced to leave from the production line to an auxiliary production line. After experiencing the period of maintenance, the machine in the production line resumes work and becomes available. Motivated by this practical application, we find that proper maintenance in queueing systems is vital to ensure the normal operation of queueing systems and reduce the incidence of the accidental failures to avoid or minimize the loss. So, in this paper, we aim to model this type of queueing systems as clearing queues with service quality feedback and analyze the strategic behavior of the customers in queueing systems.

Actually, in recent years, the study of queueing models with removals of customers before being served is a recent endeavor. One typical type of such a situation often appears in queueing systems that are subject to disasters or catastrophes. In this case, the occurrence of disasters/catastrophes pushes all customers to abandon the system, and the system needs some proper maintenances. Such systems are also referred to as stochastic clearing systems. Since the introduction of stochastic clearing systems, there has been considerable attention to this topic, see e.g., Artalejo and Gomez-Corral [1], Giorno et al. [2], Kim and Lee [3], Jiang and Liu [4], and Jiang [5]. So far, most studies on stochastic clearing queueing systems focus on the performance measures, and few research studies of this topic concentrate on strategic behavior of customers.

During the past decades, there has been a notable interest in studying the strategic behavior of customers in queueing systems from an economic viewpoint. The study of queueing systems under a game-theoretic perspective dates back to Naor [6], in which the author studied the strategic behavior 
of customers in $\mathrm{M} / \mathrm{M} / 1$ queues, where arriving customers know the number of customers in the system and decide whether to join or balk the queue based on their surplus utilities. Edelson and Hilderbrand [7] considered the strategic behavior of customers in $\mathrm{M} / \mathrm{M} / 1$ queues by studying the corresponding unobservable case. Since then, there has been considerable attention to the strategic behavior of customers in queueing systems, interested readers may also refer to the monographs of Hassin and Haviv [8], Hassin [9], recent papers of Hassin and Roet-Green [10], Ibrahim [11], and the references therein. In particular, Economou and Kanta [12] studied the equilibrium customer strategies in an observable $\mathrm{M} / \mathrm{M} / 1$ queue with breakdowns and repairs. On the basis of Economou and Kanta [12], Li et al. [13] considered the unobservable case and give some new results. Dimitrakopoulos and Burnetas [14] investigated the equilibrium and optimal strategies in an $\mathrm{M} / \mathrm{M} / 1$ queue with the dynamic service control. $\mathrm{Xu}$ and $\mathrm{Xu}$ [15] analyzed the equilibrium strategic behavior of customers in a singleserver queue with partial failures and repairs. Yu et al. [16] studied the equilibrium strategies of an unobservable M/M/1 single-server queue with balking and delayed repairs. Then, $\mathrm{Yu}$ et al. [17] investigated the equilibrium strategies in the almost observable and almost unobservable $\mathrm{M} / \mathrm{M} / 1$ queues with partial breakdowns. More related papers on the study of customer behavior in queueing systems include Wang et al. $[18,19]$ and the references therein.

In addition, for the stochastic clearing queueing systems, some researchers also give the equilibrium analysis for this type of queueing models. For example, Boudali and Economou $[20,21]$ first considered the strategic behavior of customers in a single-server Markovian queue with disasters, where batch of customers are forced to leave the system without receiving service. Next, Economou and Manou [22] studied an $M / M / 1$ clearing system in an alternating environment. Manou et al. [23] investigated strategic customers in a transportation station, which can be modelled as a stochastic clearing system with generally distributed interservice times and varying capacity. Moreover, in [22, 23], all customers can be served in batch periodically. Ma [24] studied a Markovian clearing queueing system with setup times and obtained the balking strategies of customers. Recently, Bountali and Economou [25, 26] studied the equilibrium joining strategies of customers in batch service queueing systems, which can also be seen as a special stochastic clearing queueing system. Manou et al. [27] provided a pricing analysis in a transportation station with strategic customers by considering three cases distinguished by the level of delay information. Bountali and Economou [28] studied the strategic customer behavior in a two-stage service system with batch processing. In these papers, combining the queueing theory and game theory, the authors analyze the queueing models with the assumption that arriving customers choose whether to join or balk the system on the basis of a linear reward-cost structure and then investigated the strategic behavior of customers.

To the best of our knowledge, there is no work analyzing the equilibrium behavior of delay-sensitive customers in a service system with service quality feedback. To this end, we will analyze the queueing model from an economic viewpoint. The main contributions of the paper are as follows:

(i) In the queueing systems with service quality feedback, we aim to investigate the joining decisions of the delay-sensitive customers, and it has not been considered in the open literature.

(ii) We model this type of queueing systems as clearing queues with service quality feedback and system maintenance (during times that the system which resides in the operative state follows an Erlang distribution instead of an exponential distribution) and derive formulas for the computation of equilibrium customer strategies under the two levels of information (observable and unobservable).

(iii) We also provide some numerical examples to reveal the impacts of several parameters on the equilibrium strategies, together with some intuitive explanations.

The rest of this paper is organized as follows. In Section 2 , we give the description of the proposed queueing model. In Section 3, we study the equilibrium threshold strategies for the observable case. In Section 4, we analyze the unobservable case and derive the mixed Nash equilibrium balking strategies. Section 5 is devoted to deriving the expected social benefit per time unit for the observable and the unobservable cases. In Section 6, we provide some numerical examples to show the impact of some parameters on the behavior of the customers. We conclude the paper in Section 7.

\section{Model Description}

In this paper, we model the queueing system as a clearing queueing system with service quality feedback and system maintenance. In the following, we provide the model description. Our assumptions are partly driven by the actual problem that motivates the analysis and partly by our concern to keep the model as simple as possible. In practice, it is more common to model the queueing system as a queue with multiple servers, and a maintenance procedure is immediately triggered once the system receives a certain number of negative feedbacks. However, the model will be very complicated if we consider a certain number of negative feedbacks. For analytical simplicity, we consider a capacityconstrained queueing system and assume that a maintenance procedure is immediately triggered once the system receives one negative feedback. We think the simplified model could also bring some similar conclusions and management implications.

(i) Customers arrive according to a Poisson process with rate $\lambda$, and customers are served based on a first-come, first-served (FCFS) discipline.

(ii) Service times are independent and identically distributed (i.i.d) random variable and follow an exponential distribution with parameter $\mu$. 
(iii) At a service completion instant, a customer is asked for giving a service feedback for his/her service. If the customer is satisfied with the quality of service, the server will receive a positive feedback for its kind and considerate service; otherwise, the server will receive a negative feedback. Once the system receives a negative feedback, it undergoes a maintenance procedure immediately. To this end, we consider $\theta$ as the probability that a served customer gives a positive feedback for his/her service and consider $1-\theta$ as the probability that a served customer gives a negative feedback for his/her service. Different from the queueing system with disasters, under the considering assumption, we find that during times that the system which resides in the operative state follows an Erlang distribution instead of an exponential distribution.

(iv) The maintenance period follows an exponential distribution with parameter $\xi$. During a maintenance period, arrivals are not allowed to join the system since the system stops working during this period.

(v) We further assume that the arrival times, the service times, and the maintenance times are mutually independent.

Let $\{(N(t), I(t)), t \geq 0\}$ denote the state of the clearing queueing system at time $t$, where $N(t)$ denotes the number of customers in the system at time $t$, and $I(t)$ denotes the state of the server (0: maintenance state, 1: operative state). Then, $\{(N(t), I(t)), t \geq 0\}$ is a continuous-time Markov chain with state space

$$
\Omega=\{(n, 1), n \geq 0\} \cup\{(0,0)\} .
$$

The concrete transition rates of the clearing queueing system are given as follows:

$$
\begin{aligned}
q_{(n, 1)(n+1,1)} & =\lambda, \quad n=0,1,2, \ldots, \\
q_{(n, 1)(n-1,1)} & =\mu \theta, \quad n=1,2, \ldots, \\
q_{(n, 1)(0,0)} & =\mu(1-\theta), \quad n=1,2, \ldots, \\
q_{(0,0)(0,1)} & =\xi .
\end{aligned}
$$

In the present paper, we are interested in discussing the strategic behavior of customers when they have the option to determine whether to enter the system or not. Next, we will construct a natural reward-cost structure and analyze the queueing model from an economic viewpoint. Without loss of generality, we assume that each joining customer receives either a reward of $R_{s}$ after his/her service completion or a compensation of $R_{f}$ if he/she is forced to leave the system without receiving service. In other words, $R_{s}$ represents the added value of being served (the perceived value on the service), and $R_{f}$ is a compensation value so as to eliminate customers' dissatisfaction (i.e., the system makes up for the waiting time of customers who leave the system without receiving service). To facilitate analysis, we assume $R_{s}>R_{f}$. In addition, customers are sensitive to delays, and the delay cost is in proportion to the sojourn time in the system, which is continuously accumulated from the epoch at which he/she enters the system to the epoch at which he/she leaves the system by either the occurrence of system maintenance or the service completion. Denote by $C$ the delay cost per unit of time for each customer.

In the following sections, we will study the strategic behavior of the customers regarding their joining or balking dilemma by distinguishing the observable and the unobservable cases, i.e., the arriving customers whether they join the queue or not is based on the information available at their arrival instants. Concretely, we consider two different information cases as follows: (1) observable case: customers are allowed to observe both of the queue length and the server state upon their arrivals; (2) unobservable case: customers observe only the server state upon arrival and not the queue length.

\section{The Observable Case}

We begin our analysis by investigating the clearing queueing model under the observable case, where customers can obtain the information of the system state $(n, i)$ upon their arrivals. According to our model description, customers are not allowed to join the system while the system stays in maintenance period, i.e., he/she always balks. Therefore, we only consider the case that a customer observes the system in the operative state.

Lemma 1. In the observable clearing queueing system with service quality feedback and system maintenance, if an arriving customer finds the system at state $(n, 1)$ and decides to enter, his/her expected net benefit is

$$
S_{\mathrm{obs}}(n, 1)=\theta^{n} R_{s}+\left(1-\theta^{n}\right) R_{f}-\frac{C}{\mu} \frac{1-\theta^{n+1}}{1-\theta} .
$$

In the observable case, if the expected net benefit of an arriving customer is positive, he/she prefers to join the system; if the expected net benefit of an arriving customer is zero, he/she is indifferent between entering and balking; and if the expected net benefit of an arriving customer is negative, he/she decides to balk. According to the three cases, we can conclude the following results.

Theorem 1. In the observable clearing queueing system with service quality feedback and system maintenance, there, respectively, exists a unique individual optimal pure strategy for the following three cases:

Case 1: $R_{s}<(C / \mu)$. Then, the unique individual optimal strategy is always to balk.

Case 2: $R_{s} \geq(C / \mu)$ and $R_{f}<(C / \mu(1-\theta))$. Then, the unique individual optimal strategy is the threshold strategy having the form while arriving at time $t$ and finding the system in the operative state, observe $N(t)$; join the system if $N(t) \leq n_{e}$ and balk otherwise, where $n_{e}=\left\lfloor n^{*}\right\rfloor$ and 


$$
n^{*}=\frac{\ln K}{\ln \theta}
$$

with

$$
K=\frac{(C /(1-\theta) \mu)-R_{f}}{R_{s}-R_{f}+(C \theta /(1-\theta) \mu)} .
$$

Case 3: $R_{f} \geq(C / \mu(1-\theta))$. Then, the unique individual optimal strategy is always to join the system.

Remark 1. The argument in Theorem 1 implies that the joining/balking strategy of an arriving customer is an individually optimal decision, which is independent of the strategies of all other customers. This means that the expected net benefit of a customer is not affected by the strategies of the future customers. In the game theory, the strategy obtained in this section is called dominant since it is the best response against any strategy of the others.

\section{The Unobservable Case}

We next consider the unobservable case. In this case, the arriving customers observe only the server state upon arrival and not the queue length. In order to obtain the strategic behavior of customers in the unobservable case, we first give a lemma to show the expected net benefit of a customer if he/ she decides to join the system.

Lemma 2. In the unobservable clearing queueing system with service quality feedback and system maintenance, an arriving customer finds the server operative, and the others follow a strategy q; if he/she decides to enter, his/her expected net benefit is

$$
S_{\mathrm{un}}(1, q)=\left(R_{s}-R_{f}+\frac{C \theta}{(1-\theta) \mu}\right) \frac{1-x_{2}(q)}{1-\theta x_{2}(q)}+R_{f}-\frac{C}{(1-\theta) \mu},
$$

where $x_{2}(q)$ is given by

$$
x_{2}(q)=\frac{(\lambda q+\mu)-\sqrt{(\lambda q+\mu)^{2}-4 \lambda q \mu \theta}}{2 \mu \theta} .
$$

Based on the aforementioned discussions, we can derive the Nash equilibrium joining strategies of an arriving customer in the unobservable case.

Theorem 2. In the unobservable clearing queueing system with service quality feedback and system maintenance, there exists a unique Nash equilibrium mixed strategy $q_{e}$, which has the following form:

$$
q_{e}=\left\{\begin{array}{l}
0, R_{s} \leq \frac{C}{\mu}, \\
\frac{\left(C-\mu R_{s}\right)\left[\theta C+(1-\theta)\left(\mu R_{s}-\mu R_{f}\right)\right]}{\lambda\left[C-(1-\theta) \mu R_{f}\right]\left[(1-\theta) R_{f}-R_{s}\right]}, \\
\frac{C}{\mu}<R_{s}<\frac{1}{1-x_{2}}\left(\frac{C}{\mu}-R_{f}(1-\theta) x_{2}\right), \\
1, R_{s} \geq \frac{1}{1-x_{2}}\left(\frac{C}{\mu}-R_{f}(1-\theta) x_{2}\right),
\end{array}\right.
$$

where $x_{2}=\left((\lambda+\mu)-\sqrt{(\lambda+\mu)^{2}-4 \lambda \mu \theta}\right) / 2 \mu \theta$.

Remark 2. The Nash equilibrium strategies obtained in this section are independent of the parameter $\xi$ since customers only make their joining decisions while the system resides in the operative state. Theorem 2 also reveals that, for very low values of $R_{s}$, all customers balk to the system, for very high values of $R_{s}$, all customers join to the system, while for intermediate values of $R_{s}$, customers join the system with a mixed strategy $q_{e} \in(0,1)$. Since $S_{\text {un }}(1, q)$ is a decreasing function in $q$, this means that, as more customers join the queueing system, the tagged customer is less willing to join the queue, which can be referred as avoid the crowd (ATC) situation.

\section{The Social Benefits}

In this section, we turn our attention to a social planner's point of view, i.e., we concentrate our analysis on maximizing the expected social net benefit per time unit. By constructing the expected social net benefit per time unit functions $S_{\mathrm{obs}}^{\mathrm{soc}}\left(n_{s}\right)$ and $S_{\mathrm{un}}^{\mathrm{soc}}(q)$ for the observable and unobservable cases, we can obtain the optimal values $n_{\text {soc }}$ and $q_{\text {soc }}$ to maximize the expected social benefit per time unit functions $S_{\mathrm{obs}}^{\mathrm{soc}}\left(n_{s}\right)$ and $S_{\mathrm{un}}^{\mathrm{soc}}(q)$, respectively. Next, we first investigate the observable case and give the following lemma.

Lemma 3. In the observable clearing queueing system with service quality feedback and system maintenance, if all arriving customers follow a threshold strategy with threshold $n_{s}$, the stationary distribution ( $p_{\text {obs }}^{\text {soc }}(n, i):(n, i) \in\{(n, 1), n=$ $\left.\left.0,1,2, \ldots, n_{s}+1\right\} \cup\{(0,0)\}\right)$ has the following form:

$$
\begin{aligned}
p_{\mathrm{obs}}^{\mathrm{soc}}(0,0)= & \frac{\mu(1-\theta)}{\xi}\left[d_{1}\left(n_{s}\right)\left(\frac{y_{1}\left(1-y_{1}^{n_{s}}\right)}{1-y_{1}}+\frac{\lambda}{\mu} y_{1}^{n_{s}}\right)\right. \\
& \left.+d_{2}\left(n_{s}\right)\left(\frac{y_{2}\left(1-y_{2}^{n_{s}+1}\right)}{1-y_{2}} \frac{\lambda}{\mu} y_{2}^{n_{s}}\right)\right], \\
p_{\mathrm{obs}}^{\mathrm{soc}}(k, 1)= & d_{1}\left(n_{s}\right) y_{1}^{k}+d_{2}\left(n_{s}\right) y_{2}^{k}, \quad 0 \leq k \leq n_{s}+1,
\end{aligned}
$$


where

$$
\begin{aligned}
y_{1}= & \frac{(\lambda+\mu)+\sqrt{(\lambda+\mu)^{2}-4 \lambda \mu \theta}}{2 \mu \theta}, \\
y_{2}= & \frac{(\lambda+\mu)-\sqrt{(\lambda+\mu)^{2}-4 \lambda \mu \theta}}{2 \mu \theta}, \\
d_{1}\left(n_{s}\right)= & \frac{A_{2}}{A_{2} B_{1}-A_{1} B_{2}}, \\
d_{2}\left(n_{s}\right)= & \frac{A_{1}}{A_{1} B_{2}-A_{2} B_{1}},
\end{aligned}
$$

with

$$
\begin{aligned}
& A_{i}=\frac{\mu(1-\theta) y_{i}\left(1-y_{i}^{n_{s}+1}\right)+\left(\mu \theta y_{i}-\lambda\right)\left(1-y_{i}\right)}{1-y_{i}}, \quad i=1,2, \\
& B_{i}=\frac{y_{i}\left(1-y_{i}^{n_{s}+1}\right)[\mu(1-\theta)+\xi]+\xi\left(1-y_{i}\right)}{\xi\left(1-y_{i}\right)}, \quad i=1,2 .
\end{aligned}
$$

Theorem 3. In the observable clearing queueing system with service quality feedback and system maintenance, if all

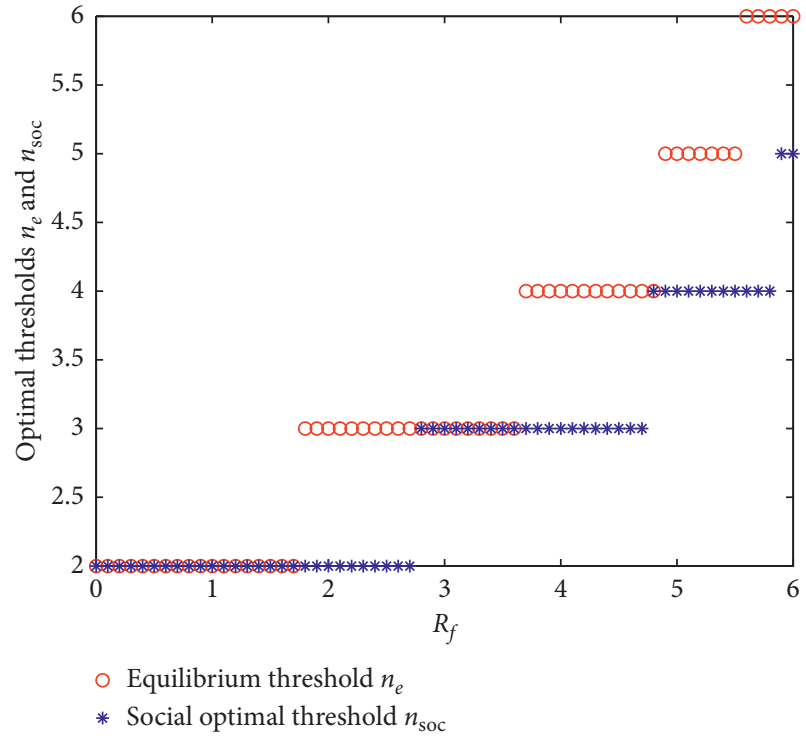

FIGURE 1: Equilibrium and social optimal thresholds versus $R_{f}$ $\left(C=3, R_{s}=7\right)$.

arriving customers adopt a threshold strategy with threshold $n_{s}$, the expected net social benefit per time unit has the following form:

$$
\begin{aligned}
S_{\mathrm{obs}}^{\mathrm{soc}}\left(n_{s}\right)= & \lambda\left(R_{s}-R_{f}\right)\left(d_{1}\left(n_{s}\right) \frac{1-\left(y_{1} \theta\right)^{n_{s}+1}}{1-y_{1} \theta}+d_{2}\left(n_{s}\right) \frac{1-\left(y_{2} \theta\right)^{n_{s}+1}}{1-y_{2} \theta}\right)+\lambda R_{f}\left(d_{1}\left(n_{s}\right) \frac{1-y_{1}^{n_{s}+1}}{1-y_{1}}+d_{2}\left(n_{s}\right) \frac{1-y_{2}^{n_{s}+1}}{1-y_{2}}\right) \\
& -C d_{1}\left(n_{s}\right)\left(\frac{y_{1}\left(1-y_{1}^{n_{s}+1}\right)}{\left(1-y_{1}\right)^{2}}-\frac{\left(n_{s}+1\right) y_{1}^{n_{s}+2}}{1-y_{1}}\right)-C d_{2}\left(n_{s}\right)\left(\frac{y_{2}\left(1-y_{2}^{n_{s}+1}\right)}{\left(1-y_{2}\right)^{2}}-\frac{\left(n_{s}+1\right) y_{2}^{n_{s}+2}}{1-y_{2}}\right),
\end{aligned}
$$

where $d_{1}\left(n_{s}\right)$ and $d_{2}\left(n_{s}\right)$ are given by Lemma 3 .

Next, we turn our attention to the unobservable case, and the corresponding expected social benefit per time unit can be derived in the following theorem.
Theorem 4. In the unobservable clearing queueing system with service quality feedback and system maintenance, if customers follow a mixed strategy with joining probability q, then, the expected net social benefit per time unit has the following form:

$$
S_{\mathrm{un}}^{\mathrm{soc}}(q)=\frac{\xi\left[\lambda q R_{s}\left(1-x_{2}(q)\right)^{2}+\lambda q R_{f} x_{2}(q)(1-\theta)\left(1-x_{2}(q)\right)-C x_{2}(q)\left(1-\theta x_{2}(q)\right)\right]}{\left(\mu(1-\theta) x_{2}(q)+\xi\right)\left(1-x_{2}(q)\right)\left(1-\theta x_{2}(q)\right)}
$$

where $x_{2}(q)$ is given by Lemma 2 .

Remark 3. From Theorems 3 and 4, we find that since the very involved form of equations (12) and (13), it is difficult to derive the maximum in closed analytic form. However, we could numerically derive some qualitative conclusions while some parameters are fixed in the numerical examples.

\section{Numerical Examples}

In this section, we explore the impacts of several parameters on the behavior of customers under the observable and unobservable cases.

First, in Figures $1-3$, we assume that $\lambda=1.5, \mu=2$, and $\theta=0.8$ and, respectively, study the influence of $R_{f}, R_{s}$, and $C$ on the equilibrium and the social optimal thresholds. From Figures 1 and 2, the equilibrium and the social optimal 


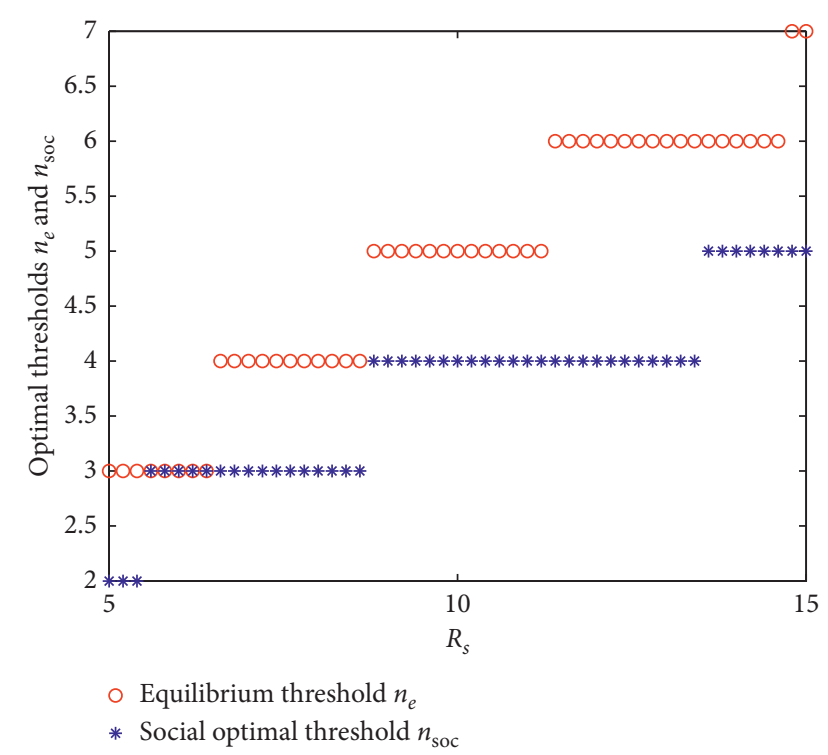

Figure 2: Equilibrium and social optimal thresholds versus $R_{s}$ $\left(C=3, R_{f}=4\right)$.

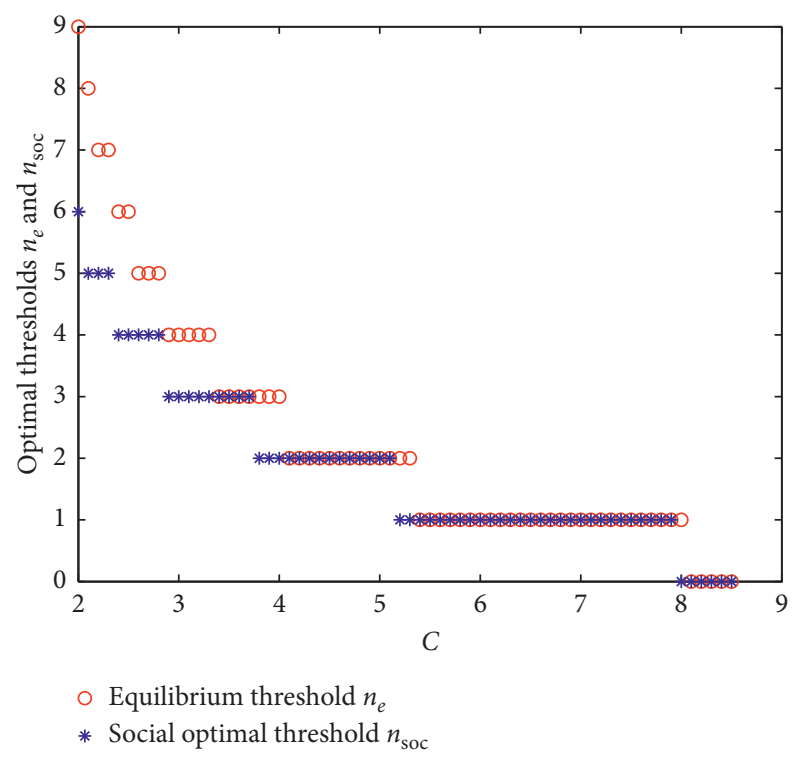

FIgURE 3: Equilibrium and social optimal thresholds versus $C$ $\left(R_{s}=8, R_{f}=4\right)$.

thresholds $n_{e}$ and $n_{\text {soc }}$ increase with the increase of $R_{f}$ and $R_{s}$, and from Figure 3, the equilibrium and the social optimal thresholds $n_{e}$ and $n_{\text {soc }}$ decrease as the waiting cost $C$ increases. It is reasonable that customers prefer to join the system if they could obtain higher value of reward (the compensation reward without receiving service or the service reward). On the contrary, customers are willing to balk to avoid paying too much if the waiting cost is high.

In Figures 4-6, we assume that $\mu=2, R_{f}=1, \theta=0.8$, and $\xi=0.8$ and pay attention to the curves of the equilibrium and the social optimal joining probabilities with the change of $R_{s}, C$, and $\lambda$. Clearly, from Figure 4, both the equilibrium and the social optimal joining probabilities are

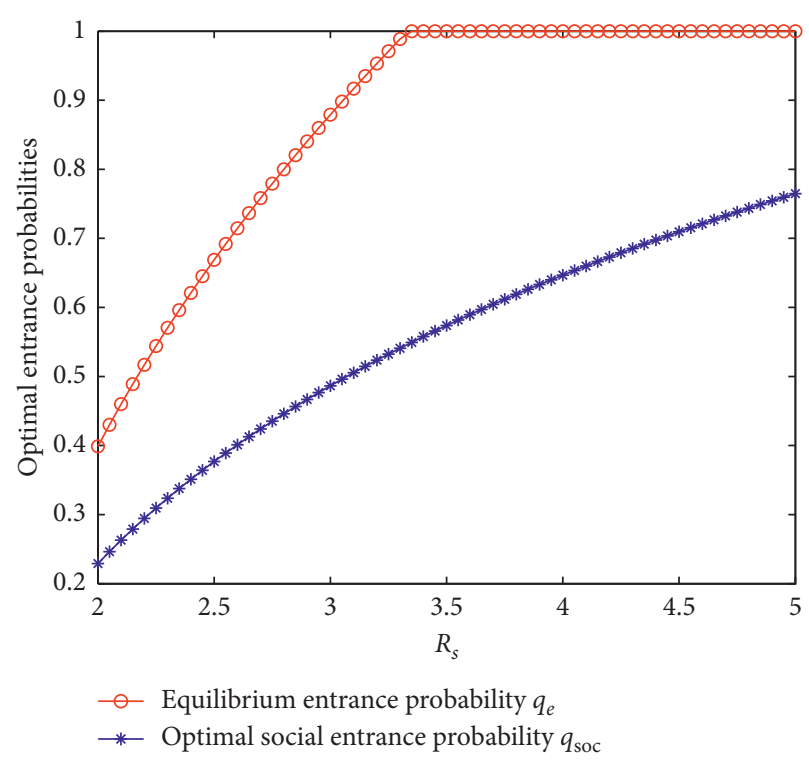

FIgURE 4: Equilibrium and social optimal joining probabilities versus $R_{s}\left(C=3, R_{f}=1, \lambda=1.5\right)$.

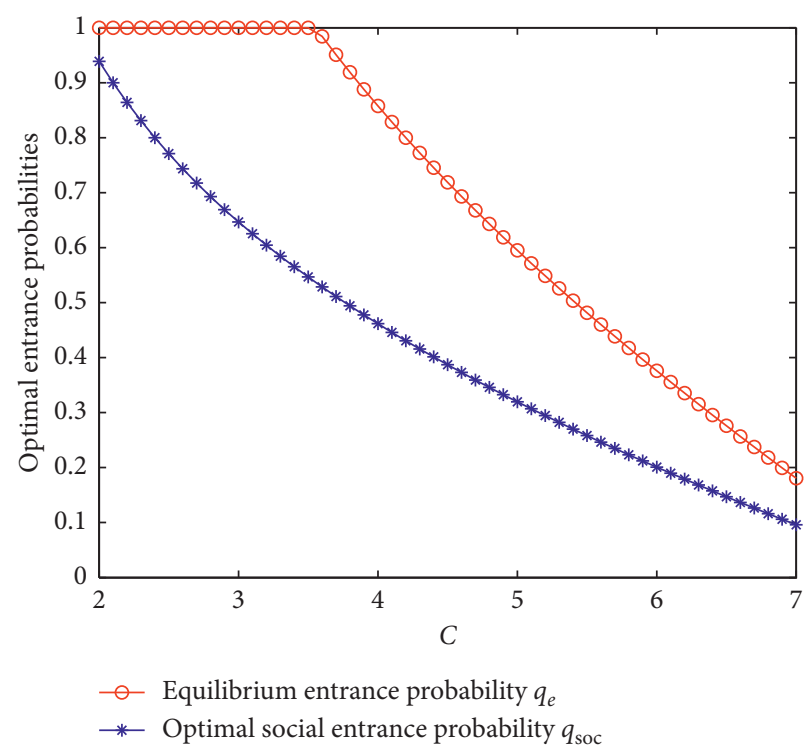

FIGURE 5: Equilibrium and social optimal joining probabilities versus $C\left(R_{s}=4, R_{f}=1, \lambda=1.5\right)$.

increasing in $R_{s}$. This is because that when $R_{s}$ increases, customers are willing to enter so as to gain more reward from service. From Figures 5 and 6, both the equilibrium and the social optimal joining probabilities are decreasing in $C$ and $\lambda$. The reason for Figure 6 is that, as $\lambda$ increases, arriving customers who find the system in the operative state predict that the system is more loaded and less tend to join the system. We could also conjecture that both the equilibrium and the social optimal joining probabilities are continuously increasing with respect to $\mu$ since the increase of service rate can benefit the customers in the system by reducing their expected waiting time. In other words, the increase of service rate can make more customers receive 


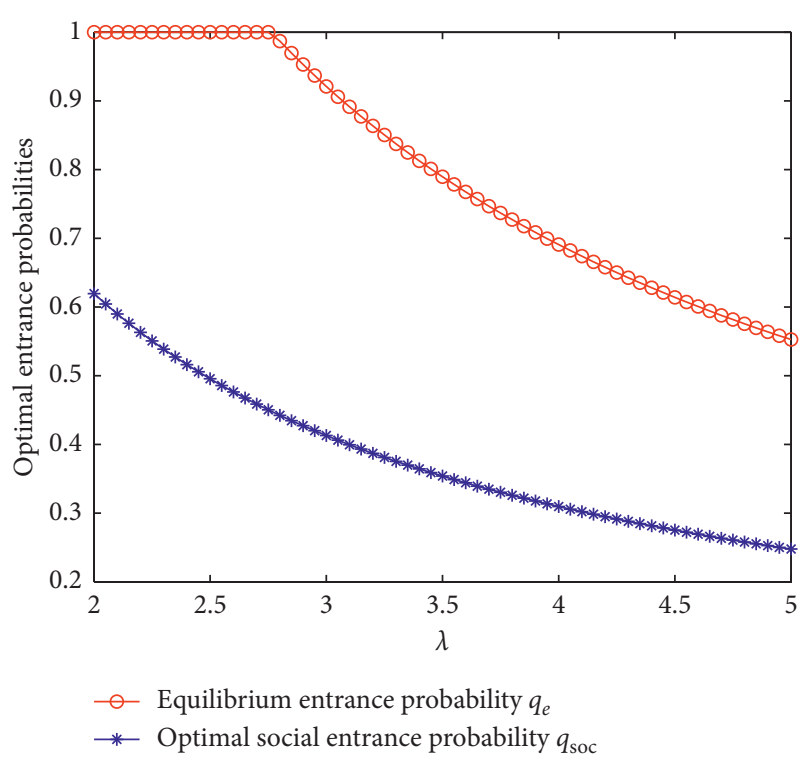

Figure 6: Equilibrium and social optimal joining probabilities versus $\lambda\left(C=2, R_{s}=4, R_{f}=1\right)$.

service which leads to arriving customers preferring to join the system.

Moreover, from Figures 1-3, we observe that $n_{\mathrm{soc}} \leq n_{e}$ for all values of $R_{f}, R_{s}$, and $C$, and from Figures 4-6, we observe that $q_{\mathrm{soc}} \leq q_{e}$ for all values of $R_{s}, C$, and $\lambda$. The reason for the results is that arriving customers intend to maximize their own benefit. When arriving customers join the system, they will increase the waiting time for future customers, i.e., they will impose negative externality effects on future customers. However, when intending to maximize the social benefit, we should take these negative externality effects into consideration. Actually, the results also hold for other values of the parameters and more general queueing models, the interested readers can refer to Boudali and Economou [20], Xu and $\mathrm{Xu}$ [15], and Wang and Zhang [29].

\section{Conclusion and Further Work}

In this paper, we studied the customer strategic behavior in a service system with service quality feedback and system maintenance. We, respectively, obtained the equilibrium threshold strategies in the observable case and the mixed Nash equilibrium balking strategies in the unobservable case. The social optimal strategies for the two cases are also derived. Finally, we illustrated some numerical examples to make a comparison between equilibrium strategies and the social optimal strategies. Although in this paper, for analytical simplicity, we just considered the case that the system undergoes an adjustment period while it receives a negative feedback. In the further work, we could also analyze the strategic behavior of customers for the case that the system undergoes an adjustment period after receiving a certain number of negative feedbacks, and it will be an interesting direction for future research. Another interesting direction for future research is that of information heterogeneity in the queue. The pricing strategy of the service provider based on customers' information heterogeneity and the impact of information heterogeneity on throughput and social welfare maximization could be an interesting research direction.

\section{Appendix}

In this section, we provide the proofs of the results in Sections 3-5.

\section{A. Proof of Lemma 1}

Proof. First, we consider a tagged customer who finds the system residing in state $(n, 1)$ upon arrival and determines to join the system. Therefore, there are $n+1$ customers in the system, and the tagged customer is in position $n+1$. Next, we consider two cases which lead the tagged customer to leave the system. Without loss of generality, we define the sojourn time of an arbitrary customer as the overall time from the epoch at which he/she joins the system to the epoch at which he/she leaves by either the service completion or the occurrence of system maintenance.

Case 1: the tagged customer may leave the system due to his/her service completion, i.e., all customers ahead of the tagged customer are satisfied with their service and give positive feedbacks. Then, in this case, the tagged customer leaves the system with probability $\theta^{n}$, and the expected sojourn time of this tagged customer is $(n+1) / \mu$.

Case 2: the tagged customer may leave the system before receiving service, i.e., there exists a customer in front of the tagged customer who is unsatisfied with his/her service. Assume that this customer is in position $j, 1 \leq j \leq n$, and he/she is the first customer to be unsatisfied with the service after the tagged customer's arrival. Then, in this case, the tagged customer leaves the system with probability $(1-\theta) \theta^{j-1}$, and his/her expected sojourn time is $j / \mu, 1 \leq j \leq n$.

Based on the reward-cost structure, for an arriving customer, if he/she observes the system at state $(n, 1)$ upon arrival and decides to join the system, his/her expected net reward is

$$
S_{\mathrm{obs}}(n, 1)=\theta^{n}\left(R_{s}-C \frac{n+1}{\mu}\right)+\sum_{k=0}^{n-1}(1-\theta) \theta^{k}\left(R_{f}-C \frac{k+1}{\mu}\right) .
$$

After some calculations, we have

$$
S_{\text {obs }}(n, 1)=\theta^{n} R_{s}+\left(1-\theta^{n}\right) R_{f}-\frac{C}{\mu} \frac{1-\theta^{n+1}}{1-\theta} \text {. }
$$

\section{B. Proof of Theorem 1}

Proof. If a tagged customer observes that the system is in state $(n, 1)$ upon arrival and makes a decision to enter, his/ her expected net benefit is given by equation (3). Simplifying (3), we have 


$$
\begin{aligned}
S_{\mathrm{obs}}(n, 1) & =\theta^{n} R_{s}+\left(1-\theta^{n}\right) R_{f}-\frac{C}{\mu} \frac{1-\theta^{n+1}}{1-\theta} \\
& =\theta^{n}\left(R_{s}-R_{f}+\frac{C \theta}{(1-\theta) \mu}\right)+R_{f}-\frac{C}{(1-\theta) \mu} .
\end{aligned}
$$

According to our assumption $R_{s}>R_{f}$, we have

$$
\frac{\mathrm{d} S_{\mathrm{obs}}(n, 1)}{\mathrm{d} n}=\left(R_{s}-R_{f}+\frac{C \theta}{(1-\theta) \mu}\right) \theta^{n} \ln \theta<0,
$$

that is, $S_{\mathrm{obs}}(n, 1)$ is a decreasing function in $n$. Hence, an arriving customer balks if $S_{\text {obs }}(n, 1)<0$; otherwise, he/she prefers to join the system. Next, we consider three cases.

If $S_{\text {obs }}(0,1)<0$, i.e., $R_{s}<(C / \mu)$, then, an arriving customer always balks. We can conclude with Case 1 .

If $S_{\text {obs }}(0,1) \geq 0$ and if $R_{f}<(C / \mu(1-\theta))$, then, there exists a unique root $n^{*}$ such that $S_{\text {obs }}\left(n^{*}, 1\right)=0$. Solving this equation, we have $n^{*}=(\ln K / \ln \theta)$, where

$$
K=\frac{(C /(1-\theta) \mu)-R_{f}}{R_{s}-R_{f}+(C \theta /(1-\theta) \mu)} .
$$

Since $S_{\mathrm{obs}}(n, 1)$ is a decreasing function in $n$, the arriving customer determines to enter if and only if $n \leq n_{e}\left(=n^{*}\right)$, and balk otherwise. Then, we can conclude with Case 2.

If $R_{f} \geq(C / \mu(1-\theta))$, then, $S_{\text {obs }}(n, 1) \geq 0$ for all $n$, and so, in this case, an arriving customer always prefers to enter into the system. We can conclude with Case 3.

\section{Proof of Lemma 2}

Proof. First, assume that each arriving customer joins the system with probability $\mathrm{q}$, and the effective arrival rate is $\lambda q$. Let $\left(p_{(n, i)}(q):(n, i) \in \Omega\right)$ denote the stationary distribution of the system. In what follows, we will refer to $p_{(n, i)}(q)$ as $p(n, i)$. Then, the steady state equations of the queueing system and the normalization condition can be driven as follows:

$$
\begin{gathered}
\xi p(0,0)=\mu(1-\theta) \sum_{k=1}^{\infty} p(k, 1), \\
\lambda q p(0,1)=\mu \theta p(1,1)+\xi p(0,0), \\
(\lambda q+\mu) p(k, 1)=\mu \theta p(k+1,1)+\lambda q p(k-1,1), \quad k \geq 1,
\end{gathered}
$$

$$
1=p(0,0)+\sum_{k=0}^{\infty} p(k, 1)
$$

It is obvious that (A.8) can be regarded as a homogeneous linear difference equation of order 2 with constant coefficients, and its corresponding characteristic equation is

$$
(\lambda q+\mu) x=\mu \theta x^{2}+\lambda q
$$

which has two roots, $x_{1}(q)$ and $x_{2}(q)$, given by

$$
\begin{aligned}
& x_{1}(q)=\frac{(\lambda q+\mu)+\sqrt{(\lambda q+\mu)^{2}-4 \lambda q \mu \theta}}{2 \mu \theta}, \\
& x_{2}(q)=\frac{(\lambda q+\mu)-\sqrt{(\lambda q+\mu)^{2}-4 \lambda q \mu \theta}}{2 \mu \theta} .
\end{aligned}
$$

Using the standard theory of homogeneous linear difference equations, we have

$$
p(k, 1)=c_{1}(q)\left[x_{1}(q)\right]^{k}+c_{2}(q)\left[x_{2}(q)\right]^{k}, \quad k \geq 0,
$$

where $c_{1}(q)$ and $c_{2}(q)$ are constants which need to be determined. Note that $x_{1}(q)>1$; hence, $c_{1}(q)$ should be necessarily equal to 0 . So, $p(k, 1)$ can be reduced to

$$
p(k, 1)=c_{2}(q)\left[x_{2}(q)\right]^{k}, \quad k \geq 0 .
$$

Based on the normalization condition, we have

$$
c_{2}(q)=\frac{\left[1-x_{2}(q)\right] \xi}{\mu(1-\theta) x_{2}(q)+\xi} .
$$

Substituting (A.14) into (A.6) and (A.13), respectively, we have

$$
\begin{aligned}
& p(0,0)=\frac{\mu(1-\theta)}{\xi} \frac{c_{2}(q) x_{2}(q)}{1-x_{2}(q)}=\frac{\mu(1-\theta) x_{2}(q)}{\mu(1-\theta) x_{2}(q)+\xi}, \\
& p(k, 1)=c_{2}(q)\left[x_{2}(q)\right]^{k}=\frac{\left[1-x_{2}(q)\right] \xi\left[x_{2}(q)\right]^{k}}{\mu(1-\theta) x_{2}(q)+\xi}, \quad k \geq 0 .
\end{aligned}
$$

When all other customers adopt the same mixed strategy with joining probability $q$, the expected net benefit of an arriving customer making a decision to join the system can be calculated by conditioning on the state that he/she observes upon arrival. The probability that the arriving customer finds $k$ customers in the system upon his/her arrival, given that he/she finds the server operative, is

$$
p^{\mathrm{un}}(k, 1)=\frac{p(k, 1)}{\sum_{j=0}^{\infty} p(j, 1)}=\left[1-x_{2}(q)\right]\left[x_{2}(q)\right]^{k}, \quad k \geq 0 .
$$

Then, if an arriving customer determines to join the system given that he/she observes that the system is in the operative state and all other customers adopt the strategy $q$, his/her expected net benefit is given by 


$$
\begin{aligned}
S_{\mathrm{un}}(1, q)= & \sum_{k=0}^{\infty} p^{\mathrm{un}}(k, 1) S_{\mathrm{obs}}(k, 1) \\
= & \sum_{k=0}^{\infty}\left[1-x_{2}(q)\right]\left[x_{2}(q)\right]^{k}\left(\theta^{k} R_{s}+\left(1-\theta^{k}\right) R_{f}\right. \\
& \left.-\frac{C}{\mu} \frac{1-\theta^{k+1}}{1-\theta}\right) \\
= & \sum_{k=0}^{\infty}\left[1-x_{2}(q)\right]\left[x_{2}(q)\right]^{k}\left(\theta^{k}\left(R_{s}-R_{f}+\frac{C \theta}{(1-\theta) \mu}\right)\right. \\
& \left.+R_{f}-\frac{C}{(1-\theta) \mu}\right) \\
= & \left(R_{s}-R_{f}+\frac{C \theta}{(1-\theta) \mu}\right) \frac{1-x_{2}(q)}{1-\theta x_{2}(q)}+R_{f}-\frac{C}{(1-\theta) \mu} .
\end{aligned}
$$

So, we obtain (6).

\section{Proof of Theorem 2}

Proof. First, differentiating $x_{2}(q)$ with respect to q, we have $\frac{\mathrm{d} x_{2}(q)}{\mathrm{d} q}=\frac{\lambda}{2 \mu \theta}\left(1-\frac{\lambda+\mu(1-\theta)-\mu \theta}{\sqrt{(\lambda+\mu(1-\theta)-\mu \theta)^{2}+4 \mu^{2} \theta(1-\theta)}}\right)>0$.

Then, $x_{2}(q)$ is strictly increasing for $q \in[0,1]$, and $S_{\text {un }}(1, q)$ is a decreasing function on $q$ since $R_{s}>R_{f}$. Define $x_{2}(0)=0, x_{2}(1)=x_{2}$; we next discuss the following three cases:

(1) If $S_{\text {un }}(1,0) \leq 0$, i.e., $R_{s} \leq(C / \mu)$, then $S_{\text {un }}(1, q)$ is nonpositive for every $q$, the best response of an arriving customer is balking, and the unique equilibrium point is $q_{e}=0$, which gives the first branch of (8).

(2) If $S_{\text {un }}(1,0)>0 \quad$ and $\quad S_{\text {un }}(1,1)<0$, i.e., $(C / \mu)<R_{s}<\left(1 / 1-x_{2}\right)\left((C / \mu)-R_{f}(1-\theta) x_{2}\right)$, there exists a unique solution of $S_{\text {un }}(1, q)=0$, which lies in $(0,1)$. Solving this equation, we obtain the second branch of (8).

(3) If $S_{\text {un }}(1,1) \geq 0$, i.e., $\quad R_{s} \geq\left(1 / 1-x_{2}\right)\left((C / \mu)-R_{f}\right.$ $\left.(1-\theta) x_{2}\right)$, then $S_{\text {un }}(1, q)$ is nonnegative for every $q$, and the best response is 1 . Thus, entering is the unique Nash equilibrium strategy, and the third branch of (8) is obtained.

\section{E. Proof of Lemma 3}

Proof. In the observable case, the corresponding stationary distribution under a threshold strategy with threshold $n_{s}$ can be obtained by solving the following steady state equations:

$$
\begin{aligned}
& \xi p_{\mathrm{obs}}^{\mathrm{soc}}(0,0)=\mu(1-\theta) \sum_{k=1}^{n_{s}+1} p_{\mathrm{obs}}^{\mathrm{soc}}(k, 1), \\
& \lambda p_{\mathrm{obs}}^{\mathrm{soc}}(0,1)=\mu \theta p_{\mathrm{obs}}^{\mathrm{soc}}(1,1)+\xi p_{\mathrm{obs}}^{\mathrm{soc}}(0,0), \\
& (\lambda+\mu) p_{\mathrm{obs}}^{\mathrm{soc}}(k, 1)=\mu \theta p_{\mathrm{obs}}^{\mathrm{soc}}(k+1,1)+\lambda p_{\mathrm{obs}}^{\mathrm{soc}}(k-1,1), \\
& \quad 1 \leq k \leq n_{s}, \\
& \mu p_{\mathrm{obs}}^{\mathrm{soc}}\left(n_{s}+1,1\right)=\lambda p_{\mathrm{obs}}^{\mathrm{soc}}\left(n_{s}, 1\right) . \\
& \text { From equation }(\mathrm{A} .21), \text { we have } \\
& p_{\mathrm{obs}}^{\mathrm{soc}}(k+1,1)-y_{1} p_{\mathrm{obs}}^{\mathrm{soc}}(k, 1) \\
& =y_{2}\left(p_{\mathrm{obs}}^{\mathrm{soc}}(k, 1)-y_{1} p_{\mathrm{obs}}^{\mathrm{soc}}(k-1,1)\right), \quad 1 \leq k \leq n_{s},
\end{aligned}
$$

which can be rewritten as the following form by considering equation (A.22), i.e.,

$$
\begin{gathered}
p_{\mathrm{obs}}^{\mathrm{soc}}(k, 1)-y_{1} p_{\mathrm{obs}}^{\mathrm{soc}}(k-1,1)=\frac{\lambda-\mu y_{1}}{\lambda} p_{\mathrm{obs}}^{\mathrm{soc}}\left(n_{s}+1,1\right) y_{2}^{n-1-n_{s}}, \\
1 \leq k \leq n_{s},
\end{gathered}
$$

where

$$
\begin{aligned}
y_{1}= & \frac{(\lambda+\mu)+\sqrt{(\lambda+\mu)^{2}-4 \lambda \mu \theta}}{2 \mu \theta}, \\
y_{2}= & \frac{(\lambda+\mu)-\sqrt{(\lambda+\mu)^{2}-4 \lambda \mu \theta}}{2 \mu \theta}, \\
d_{1}\left(n_{s}\right)= & \frac{A_{2}}{A_{2} B_{1}-A_{1} B_{2}}, \\
d_{2}\left(n_{s}\right)= & \frac{A_{1}}{A_{1} B_{2}-A_{2} B_{1}} .
\end{aligned}
$$

Simplifying equation (A.24), we have

$$
\begin{gathered}
p_{\mathrm{obs}}^{\mathrm{soc}}(k, 1)+\frac{\lambda-\mu y_{1}}{\lambda\left(y_{1}-y_{2}\right) y_{2}^{n_{s}}} p_{\mathrm{obs}}^{\mathrm{soc}}\left(n_{s}+1,1\right) y_{2}^{k} \\
=y_{1}\left(p_{\mathrm{obs}}^{\mathrm{soc}}(k-1,1)+\frac{\lambda-\mu y_{1}}{\lambda\left(y_{1}-y_{2}\right) y_{2}^{n_{s}}} p_{\mathrm{obs}}^{\mathrm{soc}}\left(n_{s}+1,1\right) y_{2}^{k-1}\right), \\
1 \leq k \leq n_{s} .
\end{gathered}
$$

By iterating equation (A.26), we can derive

$$
p_{\mathrm{obs}}^{\mathrm{soc}}(k, 1)+Y y_{2}^{k}=y_{1}^{k}\left(p_{\mathrm{obs}}^{\mathrm{soc}}(0,1)+Y\right), \quad 1 \leq k \leq n_{s},
$$

where 


$$
Y=\frac{\lambda-\mu y_{1}}{\lambda\left(y_{1}-y_{2}\right) y_{2}^{n_{s}}} p_{\mathrm{obs}}^{\mathrm{soc}}\left(n_{s}+1,1\right)
$$

Let $d_{1}\left(n_{s}\right)=p_{\text {obs }}^{\text {soc }}(0,1)+Y$ and $d_{2}\left(n_{s}\right)=-Y$, we have

$$
p_{\mathrm{obs}}^{\mathrm{soc}}(k, 1)=d_{1}\left(n_{s}\right) x_{1}^{k}+d_{2}\left(n_{s}\right) x_{2}^{k}, \quad 0 \leq k \leq n_{s} .
$$

From equation (A.19), we can derive the expression of $p_{\text {obs }}^{\text {soc }}(0,0)$. Then, based on the normalization condition

$$
p_{\mathrm{obs}}^{\mathrm{soc}}(0,0)+\sum_{k=0}^{n_{s}+1} p_{\mathrm{obs}}^{\mathrm{soc}}(k, 1)=1
$$

and equation (A.20), we can obtain $d_{1}\left(n_{s}\right)$ and $d_{2}\left(n_{s}\right)$.

\section{F. Proof of Theorem 3}

Proof. If all arriving customers join the system following a threshold strategy with threshold $n_{s}$, the expected net reward per time unit is given by

$$
S_{\mathrm{obs}}^{\mathrm{soc}}\left(n_{s}\right)=\lambda p_{\mathrm{obs}}^{s} R_{s}+\lambda p_{\mathrm{obs}}^{c} R_{f}-C E\left[Q_{\mathrm{obs}}\right]
$$

where $p_{\mathrm{obs}}^{s}$ and $p_{\mathrm{obs}}^{c}$ are the fractions of customers leaving the system due to the service completion and the occurrence of server maintenance, respectively, and $E\left[Q_{\mathrm{obs}}\right]$ is the expected number of customers in the system. Then, we have

$$
p_{\mathrm{obs}}^{s}=\sum_{k=0}^{n_{s}} p_{\mathrm{obs}}^{\mathrm{soc}}(k, 1) \theta^{k}=\sum_{k=0}^{n_{s}}\left(d_{1}\left(n_{s}\right)\left(y_{1} \theta\right)^{k}+d_{2}\left(n_{s}\right)\left(y_{2} \theta\right)^{k}\right),
$$

$$
\begin{aligned}
p_{\mathrm{obs}}^{c}= & \sum_{k=1}^{n_{s}} p_{\mathrm{obs}}^{\mathrm{soc}}(k, 1)\left(1-\theta^{k}\right)=\sum_{k=1}^{n_{s}}\left(d_{1}\left(n_{s}\right) y_{1}^{k}+d_{2}\left(n_{s}\right) y_{2}^{k}\right) \\
& \cdot\left(1-\theta^{k}\right)
\end{aligned}
$$

$$
E\left[Q_{\mathrm{obs}}\right]=\sum_{k=0}^{n_{s}+1} k p_{\mathrm{obs}}^{\mathrm{soc}}(k, 1)=\sum_{k=0}^{n_{s}+1} k\left(d_{1}\left(n_{s}\right) y_{1}^{k}+d_{2}\left(n_{s}\right) y_{2}^{k}\right)
$$

Substituting (A.32)-(A.34) into (A.31), we can obtain the result of $S_{\mathrm{obs}}^{\text {soc }}\left(n_{s}\right)$.

\section{G. Proof of Theorem 4}

Proof. If all arriving customers join the system following a mixed strategy joining probability q, given that they find the system residing in the operative state, the expected net social benefit per time unit is given by

$$
S_{\text {un }}^{\text {soc }}(q)=\lambda q p_{\text {un }}^{s}(q) R_{s}+\lambda q p_{\text {un }}^{c}(q) R_{f}-C E\left[Q_{\text {un }}\right](q),
$$

where

$$
p_{\text {un }}^{s}(q)=\sum_{k=0}^{\infty} p(k, 1) \theta^{k}=\frac{\left[1-x_{2}(q)\right] \xi}{\left(\mu(1-\theta) x_{2}(q)+\xi\right)\left(1-\theta x_{2}(q)\right)},
$$

$$
\begin{aligned}
p_{\mathrm{un}}^{c}(q) & =\sum_{k=1}^{\infty} p(k, 1)\left(1-\theta^{k}\right) \\
& =\frac{\xi x_{2}(q)(1-\theta)}{\left(\mu(1-\theta) x_{2}(q)+\xi\right)\left(1-\theta x_{2}(q)\right)}, \\
E\left[Q_{\mathrm{un}}\right](q) & =\sum_{k=0}^{\infty} k p(k, 1)=\frac{x_{2}(q) \xi}{\left(\mu(1-\theta) x_{2}(q)+\xi\right)\left(1-x_{2}(q)\right)} .
\end{aligned}
$$

Then, $S_{\text {un }}^{\text {soc }}(q)$ can be obtained by substituting (A.36)-(A.38) into (A.35).

\section{Data Availability}

All data included in this study are available upon request by contact with the corresponding author.

\section{Conflicts of Interest}

The authors declare that there are no conflicts of interest regarding the publication of this paper.

\section{Acknowledgments}

This work was supported by the Shandong Provincial Natural Science Foundation (China) (Grant no. ZR2019BG014) and the Scientific Research Foundation of Shandong University of Science and Technology for Recruited Talents (Grant no. 2019RCJJ016).

\section{References}

[1] J. R. Artalejo and A. Gomez-Corral, "Analysis of a stochasticclearing system with repeated attempts," Communications in Statistics. Stochastic Models, vol. 14, no. 3, pp. 623-645, 1998.

[2] V. Giorno, A. G. Nobile, and S. Spina, "On some time nonhomogeneous queueing systems with catastrophes," Applied Mathematics and Computation, vol. 245, pp. 220-234, 2014.

[3] B. K. Kim and D. H. Lee, "The M/G/1 queue with disasters and working breakdowns," Applied Mathematical Modelling, vol. 38, no. 5-6, pp. 1788-1798, 2014.

[4] T. Jiang and L. Liu, "Analysis of a GI/M/1 queue in a multiphase service environment with disasters," RAIRO-Operations Research, vol. 51, no. 1, pp. 79-100, 2017.

[5] T. Jiang, "Analysis of a clearing queueing system with server maintenance after $\mathrm{N}$ negative feedbacks," Probability in the Engineering and Informational Sciences, vol. 33, no. 2, pp. 220-240, 2019.

[6] P. Naor, "The regulation of queue size by levying tolls," Econometrica, vol. 37, no. 1, pp. 15-24, 1969.

[7] N. M. Edelson and D. K. Hilderbrand, "Congestion tolls for poisson queuing processes," Econometrica, vol. 43, no. 1, pp. 81-92, 1975. 
[8] R. Hassin and M. Haviv, To Queue or Not to Queue: Equilibrium Behavior in Queueing Systems, Kluwer Academic Publishers, Boston, MA, USA, 2003.

[9] R. Hassin, Rational Queueing, CRC Press, Taylor and Francis Group, Boca Raton, FL, USA, 2016.

[10] R. Hassin and R. Roet-Green, "The impact of inspection cost on equilibrium, revenue, and social welfare in a single-server queue," Operations Research, vol. 65, no. 3, pp. 804-820, 2017.

[11] R. Ibrahim, "Sharing delay information in service systems: a literature survey," Queueing Systems, vol. 89, no. 1-2, pp. 49-79, 2018.

[12] A. Economou and S. Kanta, "Equilibrium balking strategies in the observable single-server queue with breakdowns and repairs," Operations Research Letters, vol. 36, no. 6, pp. 696699, 2008.

[13] X. Li, J. Wang, and F. Zhang, "New results on equilibrium balking strategies in the single-server queue with breakdowns and repairs," Applied Mathematics and Computation, vol. 241, pp. 380-388, 2014.

[14] Y. Dimitrakopoulos and A. N. Burnetas, "Customer equilibrium and optimal strategies in an $\mathrm{M} / \mathrm{M} / 1$ queue with dynamic service control," European Journal of Operational Research, vol. 252, no. 2, pp. 477-486, 2016.

[15] B. Xu and X. Xu, "Equilibrium strategic behavior of customers in the $M / M / 1$ queue with partial failures and repairs," $O p$ erational Research, vol. 18, no. 2, pp. 273-292, 2018.

[16] S. Yu, Z. Liu, and J. Wu, "Equilibrium strategies of the unobservable $\mathrm{M} / \mathrm{M} / 1$ queue with balking and delayed repairs," Applied Mathematics and Computation, vol. 290, pp. 56-65, 2016.

[17] S. Yu, Z. Liu, and J. Wu, "Strategic behavior in the partially observable Markovian queues with partial breakdowns," Operations Research Letters, vol. 45, no. 5, pp. 471-474, 2017.

[18] J. Wang, S. Cui, and Z. Wang, "Equilibrium strategies inM/M/ 1 priority queues with balking," Production and Operations Management, vol. 28, no. 1, pp. 43-62, 2018.

[19] J. Wang and Z. G. Zhang, "Strategic joining in an $M / M / 1$ queue with risk-sensitive customers," Journal of the Operational Research Society, vol. 69, no. 8, pp. 1197-1214, 2018.

[20] O. Boudali and A. Economou, "Optimal and equilibrium balking strategies in the single server Markovian queue with catastrophes," European Journal of Operational Research, vol. 218, no. 3, pp. 708-715, 2012.

[21] O. Boudali and A. Economou, "The effect of catastrophes on the strategic customer behavior in queueing systems," Naval Research Logistics (NRL), vol. 60, no. 7, pp. 571-587, 2013.

[22] A. Economou and A. Manou, "Equilibrium balking strategies for a clearing queueing system in alternating environment," Annals of Operations Research, vol. 208, no. 1, pp. 489-514, 2013.

[23] A. Manou, A. Economou, and F. Karaesmen, "Strategic customers in a transportation station: when is it optimal to wait?," Operations Research, vol. 62, no. 4, pp. 910-925, 2014.

[24] Q. Ma, "Analysis of a clearing queueing system with setup times," RAIRO-Operations Research, vol. 49, no. 1, pp. 67-76, 2015.

[25] O. Bountali and A. Economou, "Equilibrium joining strategies in batch service queueing systems," European Journal of Operational Research, vol. 260, no. 3, pp. 1142-1151, 2017.

[26] O. Bountali and A. Economou, "Equilibrium threshold joining strategies in partially observable batch service queueing systems," Annals of Operations Research, vol. 277, no. 2, pp. 231-253, 2019.
[27] A. Manou, P. G. Canbolat, and F. Karaesmen, "Pricing in a transportation station with strategic customers," Production and Operations Management, vol. 26, no. 9, pp. 1632-1645, 2017.

[28] O. Bountali and A. Economou, "Strategic customer behavior in a two-stage batch processing system," Queueing Systems, vol. 93, no. 1-2, pp. 3-29, 2019.

[29] J. Wang and F. Zhang, "Strategic joining in M/M/1 retrial queues," European Journal of Operational Research, vol. 230, no. 1, pp. 76-87, 2013. 